\title{
Promoting Health Literacy to Prevent Hospital Readmissions: Necessary but not Sufficient
}

\author{
Michael S. Wolf, PhD MPH' , Bruce L. Lambert, PhD², and John Hickner, MD MSc ${ }^{3}$ \\ 'Division of General Internal Medicine \& Geriatrics, Feinberg School of Medicine, Northwestern University, Chicago, IL, USA; ${ }^{2}$ Center for \\ Communication and Health, School of Communications, Northwestern University, Chicago, IL, USA; ${ }^{3}$ Department of Family Medicine, University of \\ Illinois at Chicago College of Medicine, Chicago, IL, USA.
}

J Gen Intern Med 31(5):455-7

DOI: $10.1007 / \mathrm{s} 11606-016-3658-6$

(c) Society of General Internal Medicine 2016

I $\mathrm{n}$ this issue of JGIM, an article by Bell and colleagues describes a "health literacy" intervention aimed at reducing hospital readmissions by having a pharmacist coordinate medication reconciliation, patient education, and behavioral feedback from admission through transition to home. ${ }^{1}$ The "Pharmacist Intervention for Low Literacy in Cardiovascular Disease" or PILL-CVD trial presents a novel, multifaceted strategy targeting proper medication use to prevent medicationrelated readmissions of patients who have complex, multidrug regimens. The intervention components were logically coordinated-medication reconciliation upon admission, inpatient and pre-discharge written and spoken education including the teach-back technique, and phone-based postdischarge follow-up, albeit by a non-clinician. The authors refer to this as a "health literacy" strategy, given such a targeted focus on promoting medication knowledge and subsequent competencies for safe use. The features that support calling this a health literacy intervention include the strong emphasis on evidence-based practices for communicating with patients, including a method for having patients demonstrate their understanding ('teach-back' technique) and the use of more readable, actionable written materials that offer tangible supports for learning and organizing multi-drug regimens.

But beyond these well-accepted best practices, Bell and colleagues tested a healthcare system-based intervention that sought to minimize the 'cognitive demand' placed on patients at a time when they may be most acutely compromised. The continuous nature of the intervention (beginning at admission and continuing for 4 days post-discharge) addresses what has been a long known problem: our failure to prepare patients and their families for their new, often substantially modified selfcare roles once they leave the hospital. Despite the face validity of this evidence-based design, this two-site trial found that overall, the PILL-CVD intervention did not significantly reduce unplanned healthcare events (hospital admissions or emergency department visits) within 30 days of discharge. The

Published online March 9, 2016 differences between the two hospitals could have a number of explanations, including implementation differences affecting fidelity. What the authors stress as their most intriguing finding is a significant interaction between study arm and patient literacy level, with only poorly literate patients deriving benefit. By disproportionately benefitting those with more limited skills, this health literacy intervention should be praised for reducing the known disparity caused by patient literacy. But the question we are left to ponder is why these efforts were not a strong enough lever to yield meaningful reductions in readmissions for all patients.

The strategy implemented by Bell et al. was sound, reasonably intensive, and perhaps even scalable, but certain limitations deserve emphasis. The study was constrained by problems that plague most health services research studies, including financial and practical limits on sample size and number of study sites. It is unclear to what degree the study was powered to detect a reduction in adverse healthcare events, whether it be hospitalization, emergency room admission or both combined, because their sample size estimate addressed the primary outcome of medication errors (discussed in a paper published separately). ${ }^{2}$ Regardless, their findings showed no differences whatsoever in the identified primary and secondary outcomes. The significant effect among the relatively small proportion of patients with limited literacy skills was primarily driven by a reduction in emergency department (ED) visits and not hospitalizations. As there were differences in the effectiveness of the intervention by study site, another possible explanation of the literacy effect might be imbalances in literacy level between the two medical centers, which were from very different geographic locations (Nashville and Boston).

Another thing to consider when evaluating this trial is whether the intervention was powerful enough to affect the primary and secondary outcomes - hospital readmissions and ED visits. Given the intensity of programs that have yielded improvements, such as Project RED (Re-engineered Discharge) ${ }^{3}$ and BOOST (Better Outcomes by Optimizing Safe Transitions), ${ }^{4}$ is it fair to think that a 'health literacy' intervention could reduce readmissions and ED visits?

All of the components described within PILL-CVD are clearly warranted based on what we know about effective readmissions prevention, which includes an emphasis on 
proper medication use, as well as leveraging the skills of clinical pharmacists. ${ }^{5,6}$ As a patient-directed strategy striving to educate patients and support their problem-solving regarding medications, it would have been helpful in interpreting their results if Bell and colleagues had measured more proximal outcomes. For instance, the researchers do not describe if their approach produced benefits to patients' knowledge, selfefficacy, activation, and medication-taking behavior - all likely to be key drivers of any effect on readmissions. If benefits were seen on these outcomes, it might have us ponder and revise our conceptual understanding of the patient's role in preventing readmissions. Similarly, if no changes were demonstrated in these intermediate outcomes, it would provide a useful explanation of the trials' negative results and suggest specific ways the PILL-CVD intervention could be improved. Despite three decades of research, evidence of successful health literacy interventions remains scant. ${ }^{7}$ It is essential, therefore, that such intermediary measures be included in studies so we may build on our learnings.

Nonetheless, it is critical that the type of analysis Bell and colleagues performed, examining interactions between study arm and literacy level, be included in future health literacy research. Prior trials targeting improved patient comprehension and medication use have yielded the same findings; when seeking to promote health literacy within a specified clinical context, benefits may be modest among the sample as a whole, but those who are at greater risk of an adverse outcome due to limited health literacy still benefit. ${ }^{7,8}$ This in itself is a very important message. However, the value of such a finding must also be considered while taking into account the cost a healthcare system would ultimately incur to set a PILL-CVD type intervention as a new standard of care. Even if an intervention poses no threat in terms of potential harm to patients, its benefit would be limited to a small percentage of patients.

The article by Bell et al. is provocative. Despite the trial's sound conceptual grounding and mapping of solutions to common problems in acute care pertaining to patient communication, the intervention had no significant effect overall. Clearly, elements of their intervention should be standards of care if they do not already exist within most healthcare systems. This would include an appropriate protocol for medication reconciliation at hospital entry, improved and continued health communication, and outreach post-discharge to ensure a patient's successful transition. As the PILL-CVD trial was implemented several years ago, certain components of the intervention may already be commonplace within tertiary hospital settings. Similarly, all of these elements are already present in other evidence-based readmissions prevention programs, including Project RED, which was developed and evaluated before this study. Specific health literacy components such as the teach-back technique have been mainstays for the Agency for Healthcare Research and Quality (AHRQ)funded, commercial RED program.

A lesson learned perhaps is that far greater organizational communication, hand-off, and transition support components are needed to ensure patients safely return home and adapt accordingly. From a clinical decision-making perspective, Bell and colleagues have also promoted the deintensification and simplification of prescribed drug therapies to lessen the self-care burden among patients, especially the elderly. ${ }^{9}$ Moreover, many activities described in this article involved broad care coordination and had little to do with medication management, arguably making pharmacists perhaps a less natural choice. This seems to be the major difference between PILL-CVD and Project RED, as well as other known readmissions prevention programs that have been successful. All of these effective programs include a strong "receptor" component: a healthcare professional, be it a physician, nurse, social worker or community health worker, in the outpatient setting who receives the baton handoff without dropping it. Finally, many patients likely need more than one follow-up after discharge. To this end, comprehensive approaches that could offer an algorithm for assessing and determining a patient's risk for readmission would be a helpful addition to the discharge process. Factors including but not limited to health literacy, cognition, patient activation, socioeconomic status, medication regimen complexity, functional social support, and mental health all would be candidates for consideration. Such a tool would aid health care systems not only in allocating resources to those demonstrating the most need for prevention, but also in informing the nature of remediation needed. This follows the assumption that patients and their families' transition needs are variable, and therefore no one standard response may be appropriate for all.

Overall, the PILL-CVD health literacy intervention presented by Bell and colleagues is a commendable trial from which to learn. Unfortunately, there are too many examples of laudable strategies that included reasonable, even intensive efforts to change healthcare systems that were unsuccessful. ${ }^{10}$ Solutions for addressing readmissions, or any chronic disease care management concern, will require clear health communication strategies and much more to achieve improvement.

Corresponding Author: John Hickner, MD MSc; Department of Family MedicineUniversity of Illinois at Chicago College of Medicine, 1919 W Taylor, Rm 196, MC 663, Chicago, IL 60612, USA (e-mail: hickner@uic.edu).

\section{Compliance with Ethical Standards:}

Conflict of Interest: The authors declare that they do not have a conflicts of interest.

\section{REFERENCES}

1. Bell SP, Schnipper JL, Goggins K, et al. Effect of pharmacist counseling intervention on health care utilization following hospital discharge: a randomized control trial. J Gen Intern Med. doi:10.1007/s11606-0163596-3 
2. Kripalani S, Roumie CL, Dalal AK, Cawthon C, Businger A, Eden SK, et al. Effect of a pharmacist intervention on clinically important medication errors after hospital discharge: a randomized trial. Ann Intern Med 2012;157:1-10.

3. Jack BW, Veerappa KC, Anthony D, Greenwald JL, Sanchez GM, Johnson AE, et al. A reengineered hospital discharge program to decrease rehospitalization: a randomized trial. Ann Intern Med. 2009; 150: 178-187.

4. Hansen LO, Greenwald JL, Budnitz T, Howell E, Halasyamani L, Maynard G, et al. Project BOOST: effectiveness of a multihospital effort to reduce rehospitalization. J Hosp Med. 2013;8:421-427.

5. Kansagara D, Englander H, Salanitro A, et al. Risk prediction models for hospital readmission: a systematic review. JAMA. 2011;306:1688-1698.
6. Kaboli PJ, Hoth AB, McClimon BJ, Schnipper JL. Clinical pharmacists and inpatient medical care: a systematic review. Arch Intern Med. 2006; 166:955-964.

7. Berkman ND, Sheridan SL, Dohahue KE, Crotty K. Low health literacy and health outcomes: an updated systematic review. Ann Intern Med. 2011;155:97-107.

8. Wolf MS, Davis TC, Curtis LM, Webb JA, Bailey SC, Shrank WH, et al. Effect of standardized, patient-centered label instrucdtions to improve comprehension of prescription drug use. Med Care. 2011;49:96-100.

9. Holmes HM, Min LC, Yee M, Varadhan R, Basran J, Dale W, Boyd CM. Rationalizing prescribing for older patients with multimorbidity: considering time to benefit. Drugs Aging. 2013;30:655-666.

10. Frosch DL, Uy V, Ochoa S, Mangione CM. Evaluation of a behavior support intervention for patients with poorly controlled diabetes. Arch Intern Med. 2011;171:2011-2017. 